\title{
Implications of modelling one-dimensional impact by using a spring and damper element
}

\author{
A Jönsson $^{1}$, J Bathelt ${ }^{2}$, and G Broman ${ }^{1 *}$ \\ ${ }^{1}$ School of Engineering, Blekinge Institute of Technology, Karlskrona, Sweden \\ ${ }^{2}$ Institute of Mechanics, Swiss Federal Institute of Technology, Zurich, Switzerland
}

The manuscript was received on 20 April 2004 and was accepted after revision for publication on 13 October 2004.

DOI: $10.1243 / 146441905 X 9926$

\begin{abstract}
A spring and damper contact force element is often used for modelling impact in multi-body dynamics. The related condition for transition between contact and non-contact is, however, inconsistently implemented in the literature and commercial software. This comparative study aims to clarify the implications of four commonly used transition conditions. Principal differences are discussed and, by simulation of a typical system, it is shown that there are significant differences in the dynamics of the system depending on the different conditions. Two of them give unrealistic contact forces and should imply incorrect prediction of system dynamics in most applications. This suggests that it is important to review results obtained from using these conditions and to eliminate them from commercial software. A discussion of the two other conditions culminates in a recommendation.
\end{abstract}

Keywords: contact dynamics, mechanics of machines, multi-body dynamics, non-linear dynamics, soil compaction

\section{INTRODUCTION}

Impact between mechanical bodies has been studied for a long time. (see reference [1] for a historical background). In multi-body dynamics, a linearly elastic spring in parallel with a viscous damper is often used as a contact force element for modelling impact (see, for example, references [2] to $[\mathbf{1 0}])$. The related condition for transition between contact and non-contact is, however, inconsistently implemented in the literature and commercial software (see references [3] to [5], [8], and [11]). Sometimes, a transition condition is not described at all (see references [2], [9], and [12]). This is confusing and may lead to incorrect prediction of system dynamics.

This study aims to clarify the implications of four commonly used transition conditions. Principal differences are discussed and highlighted by simulation of a typical system.

\footnotetext{
* Corresponding author: School of Engineering, Blekinge Institute of Technology, SE-371 79 Karlskrona, Sweden.
}

\section{CONTACT FORCE LAWS}

Impact between two multi-body systems can generally be described as interaction via a contact force law between the two impacting bodies, $m_{1}$ and $m_{2}$, and with the influence from the other parts of the two systems modelled as forces, $F_{1}(t)$ and $F_{2}(t)$, where $t$ is time (see Fig. 1).

A contact force law consists of a model (element) giving a force, $F_{\mathrm{f}}$, as a function of the state of this model, and a condition for transition between contact and non-contact. Generally, the contact force is

$$
F_{\mathrm{c}}= \begin{cases}F_{\mathrm{f}}, & \text { at contact } \\ 0, & \text { at no contact }\end{cases}
$$

In this study the force element is a linearly elastic spring in parallel with a viscous damper, giving

$$
F_{\mathrm{f}}=c\left(\dot{x}_{1}-\dot{x}_{2}\right)+k\left(x_{1}-x_{2}\right)
$$

where $c$ is the viscous damping and $k$ is the spring stiffness. The spring and the damper could be thought of as connected by a massless plate. This 


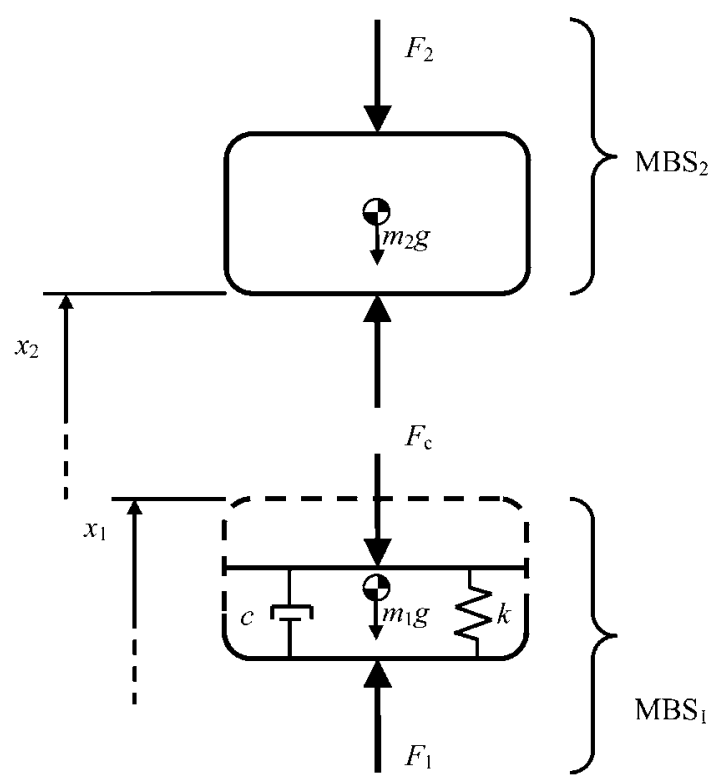

Fig. 1 General description of two impacting multibody systems

plate could be thought of as replacing the boundary of one of the impacting bodies, while the boundary of the other body is thought of as rigid, as indicated in Fig. 1, although, in reality, there will be some deformation in both bodies.

The impact can be divided into a compression phase, in which the centres of gravity of $m_{1}$ and $m_{2}$ approach each other, followed by an expansion phase, in which they depart from each other. It is assumed that the centres of gravity could be considered as fixed in relation to the undeformed boundaries of the impacting bodies. If so, the equations of motion are

$$
\begin{aligned}
& m_{1} \ddot{x}_{1}=-m_{1} g-F_{\mathrm{c}}+F_{1} \\
& m_{2} \ddot{x}_{2}=-m_{2} g+F_{\mathrm{c}}-F_{2}
\end{aligned}
$$

Integration of this equation is straightforward both at contact and non-contact. Since the state of the system influences the contact, and vice versa, the solution is, however, clearly dependent on the transition condition. In the following sections, four commonly used conditions, labelled A to D, are described and analysed. Throughout, a superscript minus sign indicates start of contact and a superscript plus sign indicates end of contact.

\subsection{Transition condition A}

It can be intuitive to use the distance between the impacting bodies as the indicator for contact. This approach can be found frequently in the literature [3-5] and in commercial software [11]. This transition condition, for both start and end of contact, is

$$
x_{1}-x_{2}=0
$$

Using equations (1) and (2) and setting $\left(x_{1}-x_{2}\right)^{-}=0$, the initial contact force becomes

$$
F_{\mathrm{c}}^{\mathrm{A}^{-}}=F_{\mathrm{f}}^{\mathrm{A}^{-}}=c\left(\dot{x}_{1}-\dot{x}_{2}\right)^{-}
$$

Since $\left(\dot{x}_{1}-\dot{x}_{2}\right)^{-}>0$ for every start of contact, this initial force is greater than zero and the discontinuity is as seen from the damping term.

Similarly, it can be shown that at the end of contact the contact force is

$$
F_{\mathrm{c}}^{\mathrm{A}^{+}}=F_{\mathrm{f}}^{\mathrm{A}^{+}}=c\left(\dot{x}_{1}-\dot{x}_{2}\right)^{+}
$$

Since $\left(\dot{x}_{1}-\dot{x}_{2}\right)^{+}<0$ for every end of contact, this implies that there is an adhesive contact force. This may be true in some special case where there is actually adhesion present. If so, adhesion should be properly included in the model. However, for the more common case where there is no or very weak adhesion, this transition condition fails in detecting the true transition from contact to noncontact and allows a fictive contact force to influence the dynamics of the multi-body system.

\subsection{Transition condition B}

A transition condition that uses the force in the force element as an indicator can also be found in the literature [8]. It states, for both start and end of contact, that

$$
F_{\mathrm{f}}^{\mathrm{B}}=0
$$

This ensures that there will never be an adhesive contact force. Furthermore, the force is continuous, because, by definition, the contact force will always be zero at the start and end of contact. Letting $F_{\mathrm{f}}^{\mathrm{B}-}=0$ and using equation (2), the displacement at the start of contact is

$$
\left(x_{1}-x_{2}\right)^{-}=-\frac{c\left(\dot{x}_{1}-\dot{x}_{2}\right)^{-}}{k}
$$

Since $\left(\dot{x}_{1}-\dot{x}_{2}\right)^{-}>0$ for every start of contact, this implies that the contact is active before the relative distance between the impacting bodies is zero. This has no physical meaning and thus this transition condition fails in detecting the true transition from noncontact to contact and allows a fictive contact force to influence the dynamics of the multi-body system. 
Similarly, it can be shown that at the end of contact

$$
\left(x_{1}-x_{2}\right)^{+}=-\frac{c\left(\dot{x}_{1}-\dot{x}_{2}\right)^{+}}{k}
$$

which is physically possible.

\subsection{Transition condition $\mathrm{C}$}

Since condition A works well at the start of contact and condition B works well at the end of contact, a combination of the two should be appropriate $[10,13,14]$. This is expressed as

$$
\left(x_{1}-x_{2}\right)^{-}=0 \wedge F_{\mathrm{f}}^{\mathrm{C}^{+}}=0
$$

In the same way as for conditions $\mathrm{A}$ and $\mathrm{B}$, it can be shown that

$$
F_{\mathrm{f}}^{\mathrm{C}^{-}}>0 \wedge\left(x_{1}-x_{2}\right)^{+}>0
$$

The principal contact behaviour for a single impact for transition conditions A to $\mathrm{C}$ is summed up in Fig. 2. Dashed lines show the differences.

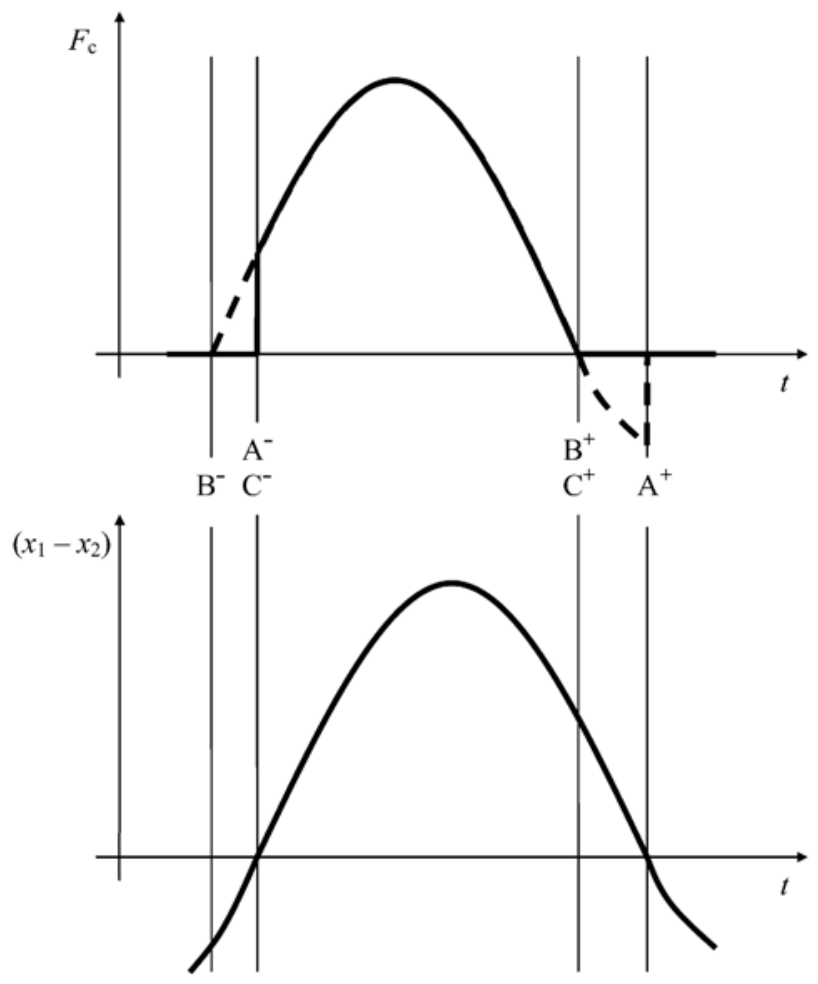

Fig. 2 Principal contact behaviour for transition conditions A to $\mathrm{C}$

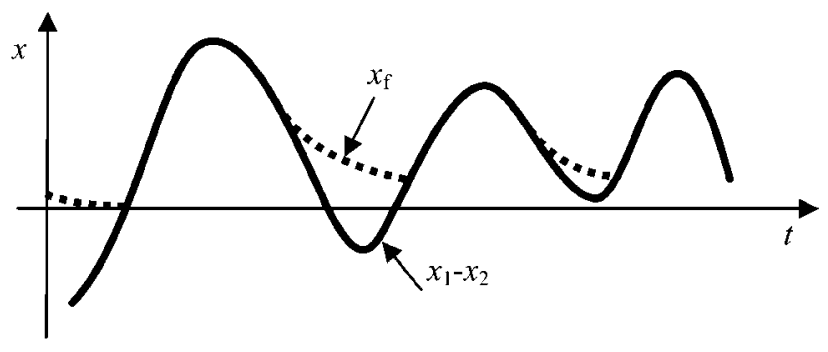

Fig. 3 Different possibilities for new contact when $x_{\mathrm{f}}>0$

\subsection{Transition condition D}

With all the above conditions, the compression of the contact force element is inherently assigned as $x_{\mathrm{f}}=x_{1}-x_{2}$. This is, however, true only at true contact. Condition $\mathrm{C}$ is thus sufficient if this compression is zero before impact and if the behaviour of the contact force element itself is of no relevance after loss of contact.

To be fully general it is, however, necessary to integrate $x_{\mathrm{f}}$ separately during non-contact from

$$
0=c \dot{x}_{\mathrm{f}}+k x_{\mathrm{f}}, \quad x_{\mathrm{f}}\left(t^{+}\right)=x_{\mathrm{f}}^{+}=\left(x_{1}-x_{2}\right)^{+}
$$

giving

$$
x_{\mathrm{f}}=x_{\mathrm{f}}^{+} \mathrm{e}^{(-k / c)\left(t-t^{+}\right)}
$$

and to modify the condition for the coming transition from non-contact to contact into

$$
\left(x_{1}-x_{2}\right)^{-}=x_{\mathrm{f}}
$$

See Fig. 3 for an illustration of principal contact possibilities. As seen, this condition does not overlook the possibility of regained contact without $\left(x_{1}-x_{2}\right)$ having passed through the zero level. The significance of this increases with increased damping and increased impact frequency.

\subsection{Summary of the contact force laws}

An overview of the principal characteristics of the studied contact force laws is given in Table 1.

\section{SIMULATION EXAMPLE}

The multi-body system in Fig. 4 is often used for the modelling of vibratory roller compaction machines $[\mathbf{8}, \mathbf{9}, \mathbf{1 5}, \mathbf{1 6}]$. It has repeatedly proven to give good agreement between measurements and simulations. 
Table 1 Principal characteristics of the studied contact force laws (bold entries indicate an active condition)

Transition conditions

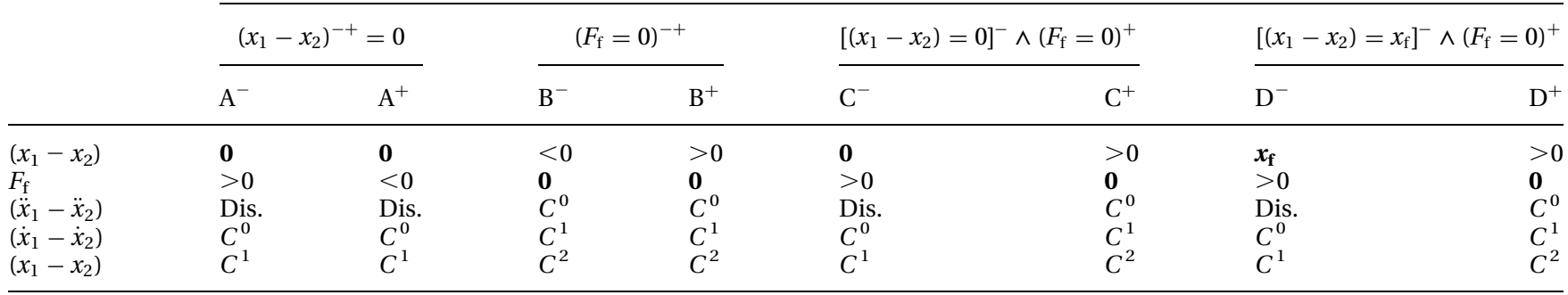

Dis. = discontinuous function; $C^{n}=$ differentiable $n$ times.

This system can be described by the following equations, combined with a contact force law

$$
\begin{aligned}
m_{2} \ddot{x}_{2}= & -m_{2} g+F_{\mathrm{c}}+c_{23}\left(\dot{x}_{3}-\dot{x}_{2}\right)+k_{23} \\
& \left(x_{3}-x_{2}-d\right)-F_{\mathrm{e}} \\
m_{3} \ddot{x}_{3}= & -m_{3} g-c_{23}\left(\dot{x}_{3}-\dot{x}_{2}\right)-k_{23}\left(x_{3}-x_{2}-d\right)
\end{aligned}
$$

where $d$ is the distance between $m_{2}$ and $m_{3}$ at which the intermediate spring is undeformed; $F_{\mathrm{c}}$ is defined in equation (1). The centre of gravity of $m_{1}$ is fixed. The excitation force is

$$
F_{\mathrm{e}}=F_{\omega} \sin (\omega t)
$$

where the amplitude $F_{\omega}$ is assumed to be constant for a given angular frequency, $\omega$, of the eccentric included in the roller.

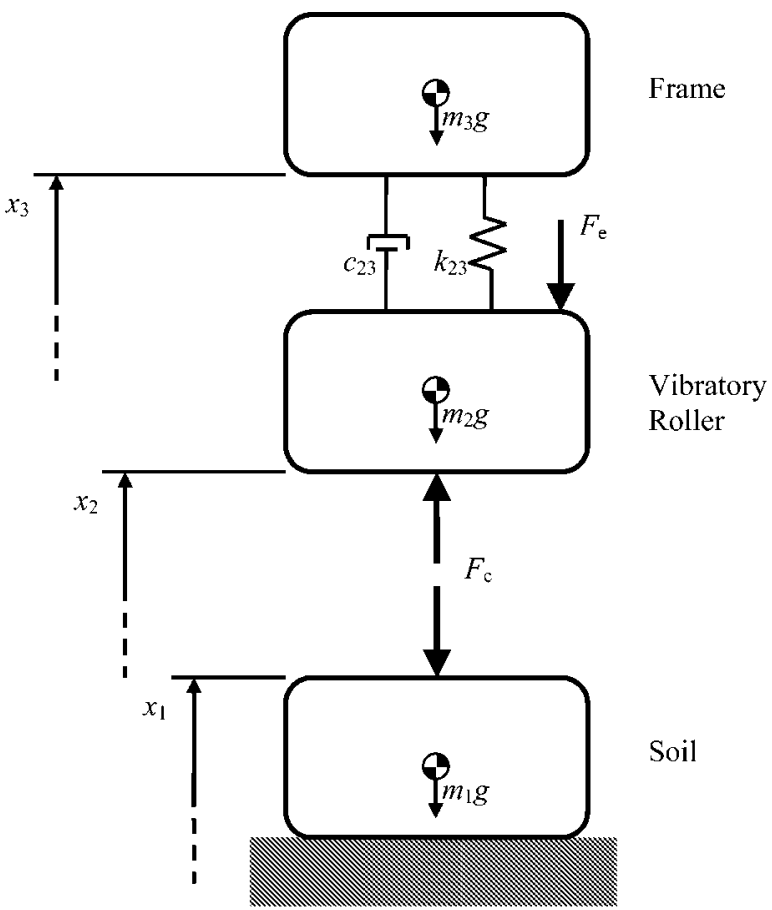

Fig. 4 Multi-body system representing a vibratory roller machine
Table 2 Parameter combinations of the simulation example

\begin{tabular}{lllll}
\hline Parameter & Case I & Case II & Case III & Case IV \\
\hline$k(\mathrm{~N} / \mathrm{m})$ & $5 \times 10^{6}$ & $2 \times 10^{8}$ & $2 \times 10^{8}$ & $2 \times 10^{8}$ \\
$D$ & 0.4 & 0.4 & 0.4 & 2.0 \\
$c(\mathrm{~N} \mathrm{~s} / \mathrm{m})$ & $8 \times 10^{4}$ & $5.06 \times 10^{5}$ & $5.06 \times 10^{5}$ & $2.53 \times 10^{6}$ \\
$\omega(\mathrm{rad} / \mathrm{s})$ & $40 \pi$ & $40 \pi$ & $80 \pi$ & $80 \pi$ \\
\hline
\end{tabular}

The system is implemented in Matlab [17] and is simulated until steady state. Interesting outputs are the displacement, velocity, and acceleration of the frame, vibratory roller, and soil.

In the numerical solution it is important to adjust the solver parameters properly. For example, if too large time steps are used, the start and end of contact would not be properly captured. Also, the location of the roller could become below the location of the compressed soil, which is, of course, not physically correct and must be prohibited.

The system is simulated for the four model parameter combinations given in Table 2, where the damping constant has been calculated according to $[13]$

$$
c=2 D \sqrt{m_{2} k}
$$

with $D$ chosen to have a value of 0.4 [18], except in the fourth combination where it is set 5 times higher.

The other parameters of the model $[\mathbf{8}]$ are $m_{2}=2 \times 10^{3} \mathrm{~kg}, m_{3}=10^{3} \mathrm{~kg}, k_{23}=10^{6} \mathrm{~N} / \mathrm{m}, c_{23}=$ $10^{4} \mathrm{~N} \mathrm{~s} / \mathrm{m}, g=9.81 \mathrm{~m} / \mathrm{s}^{2}$, and $F_{\omega}=58.8 \times 10^{3} \mathrm{~N}$.

\section{RESULTS}

\subsection{Case I}

Figure 5 shows the acceleration of the roller as a function of time at steady state. For this model parameter combination the roller $\left(m_{2}\right)$ always stays in contact with the soil (contact force element). 


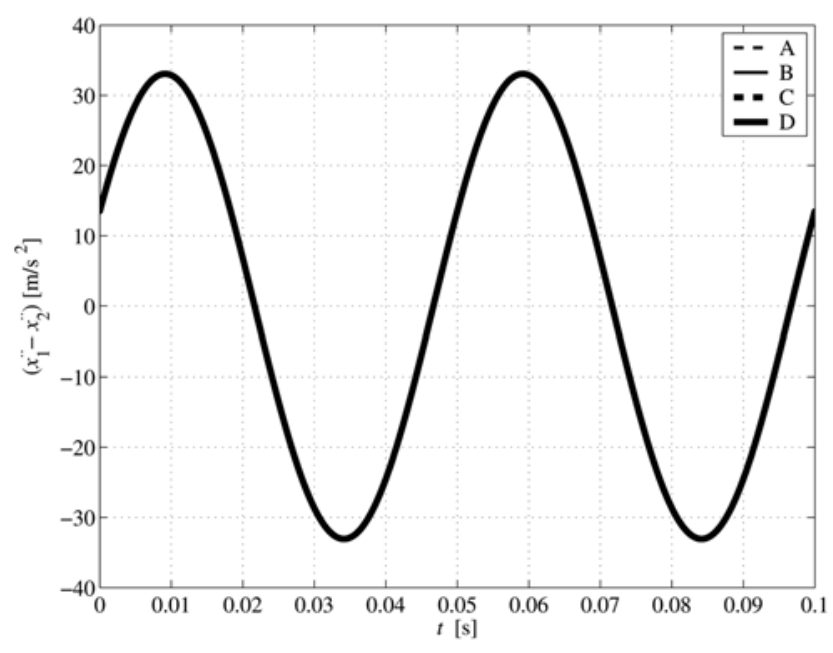

Fig. 5 Simulation results for case I

Thus, there is never any transition between contact and non-contact and consequently there is no difference in the system dynamics depending on the four different transition conditions.

\subsection{Case II}

Figure 6 shows the acceleration of the roller as a function of time at steady state. For this model parameter combination there is clearly impact. The system dynamics is essentially identical for transition conditions $\mathrm{C}$ and $\mathrm{D}$, but conditions $\mathrm{A}$ and $\mathrm{B}$ result in behaviours that differ considerably from this and also somewhat between each other.

\subsection{Case III}

Figure 7 shows the acceleration of the roller as a function of time at steady state. For this model

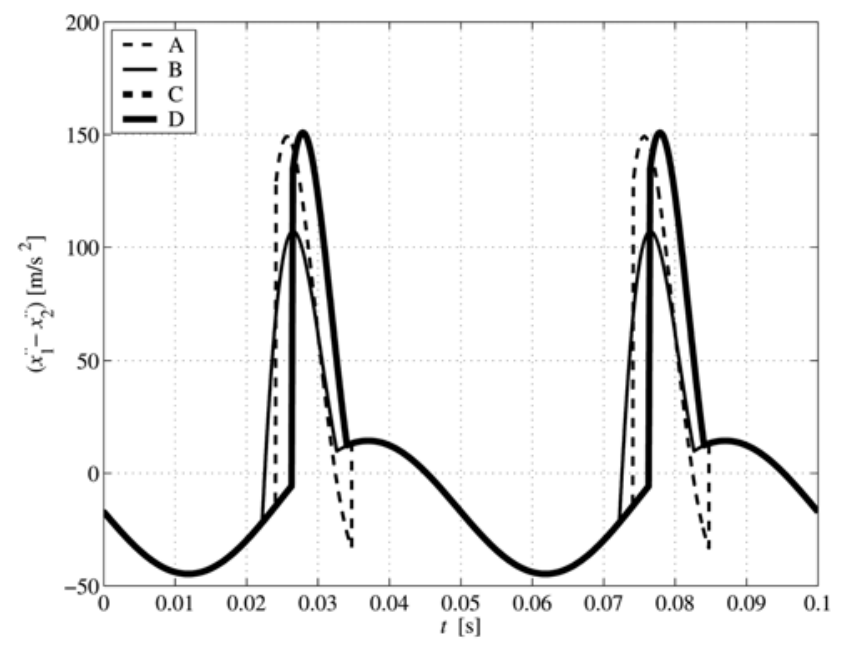

Fig. 6 Simulation results for case II

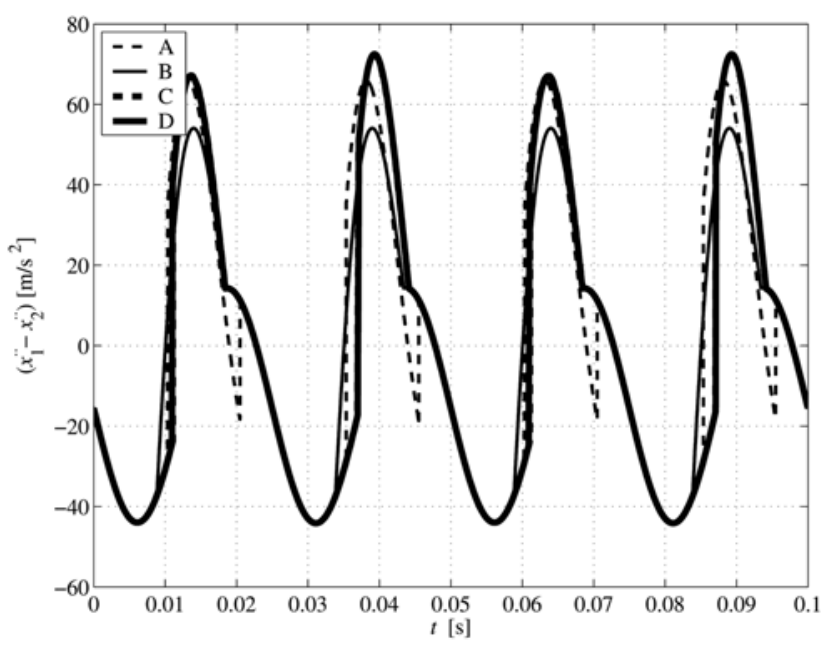

Fig. 7 Simulation results for case III

parameter combination the differences are more pronounced. Since the time for relaxation of the soil (contact force element) is shorter, because of the higher frequency, there is now some difference in the system dynamics for conditions $\mathrm{C}$ and $\mathrm{D}$, although still hardly discernible. There is also a principal difference in the system dynamics for conditions $\mathrm{A}$ and $\mathrm{B}$ compared with conditions $\mathrm{C}$ and $\mathrm{D}$. The latter now have a period 2 motion.

\subsection{Case IV}

Figure 8 shows the acceleration of the roller as a function of time at steady state. Figure 9 shows the position of the roller as a function of time at steady state. In this model parameter combination the damping has been increased significantly to illustrate more clearly the principal difference in the system dynamics for transition conditions C and D,

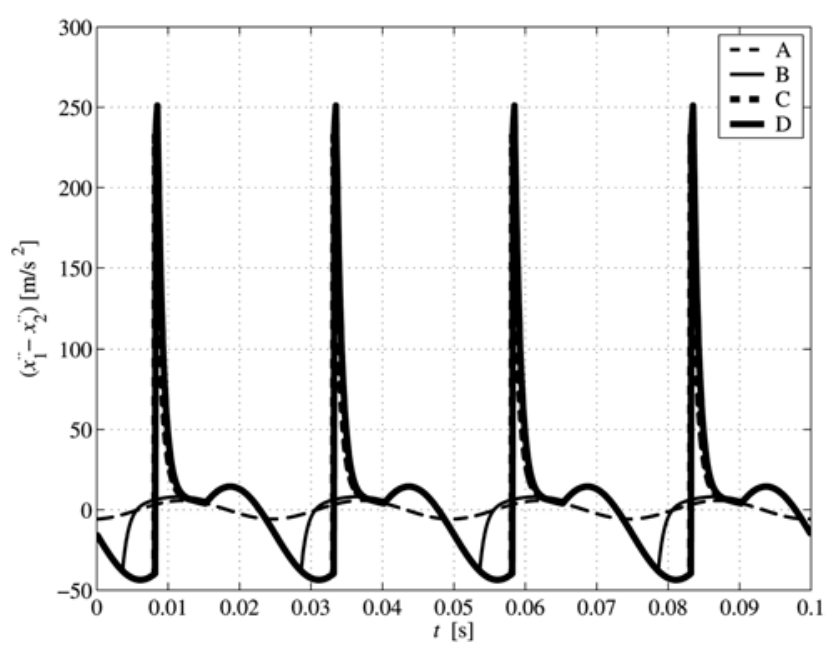

Fig. 8 Simulation results for case IV 


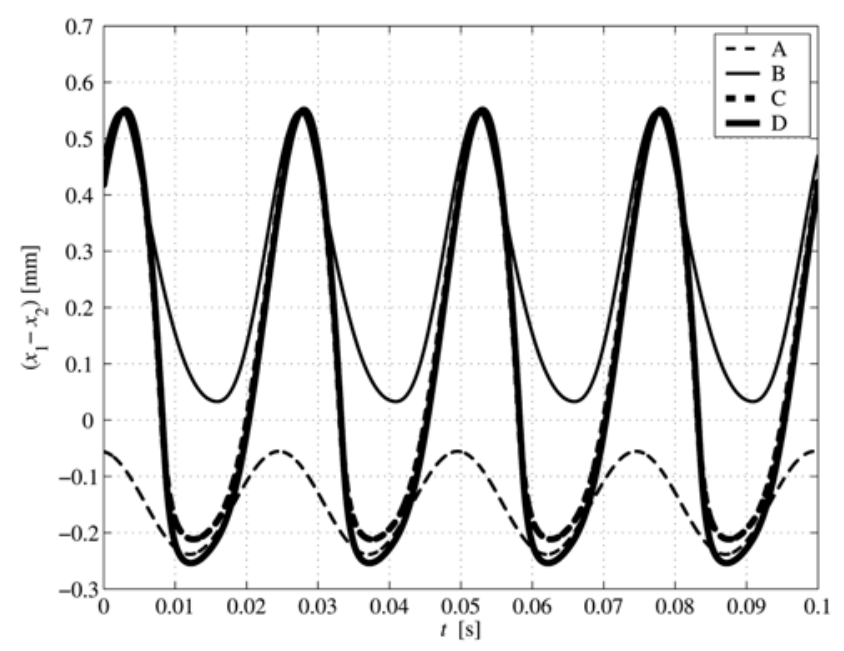

Fig. 9 Simulation results for case IV

although this damping may not be realistic for the vibratory roller on soil application.

For transition condition A there is no impact, since the adhesive contact force prevents this. Transition condition B gives a completely different and unphysical behaviour. The roller $\left(m_{2}\right)$ never touches the soil (contact force element) and yet there is a contact force. The difference in the system dynamics for conditions $\mathrm{C}$ and $\mathrm{D}$ is clearly discernible, which is due to the slow relaxation of the soil (contact force element) caused by the high damping.

\section{DISCUSSION AND CONCLUSION}

The implications of four different conditions for transition between contact and non-contact, which are commonly used together with a spring and damper contact force element when modelling impact in multi-body dynamics, are the subject of this study. The relevance of this contact force element as such in relation to various applications is not discussed.

Using (A) only the distance between the impacting bodies as the indicator [3-5] fails in detecting the true transition from contact to non-contact. Using (B) only the force in the contact force element as the indicator [8] fails in detecting the true transition from non-contact to contact. On account of this, these conditions allow a fictive contact force to influence the dynamics of the multi-body system at impact operation. With these conditions the system dynamics will thus be correctly predicted only if there is no loss of contact, that is, if there is no true impact. This is, of course, generally not known beforehand.

Both from a principal point of view and from the studied simulation example, this study suggests that it is important to review results obtained from using these conditions and to eliminate them from commercial software.

A combination of transition conditions $\mathrm{A}$ and $\mathrm{B}$, so that $\mathrm{A}$ is used to detect start of contact and $\mathrm{B}$ is used to detect end of contact, should be sufficient in many applications. To be more specific, it is suffcient if the compression of the contact force element is zero before impact and if the behaviour of the contact force element itself is of no relevance after loss of contact. This is, for example, usually the case when simulating the dynamics of compactor machines $[13,14]$. As the machine is moving horizontally at the same time as part of it performs vertical impact, this impacting part will hit 'new' soil, or the same combination of new and compacted soil, in each impact. And, of course, a solution where the impacting part would not pass above the level of uncompacted soil before the next impact could be ruled out as non-feasible for this application.

However, to be fully general, the possibility of regained contact before the compression of the contact force element has relaxed to its zero level should not be overlooked. To be able to consider this, transition condition $\mathrm{C}$ needs to be slightly modified and combined with a separate integration of the contact force element compression during non-contact (D). The significance of this increases with increased damping and increased impact frequency. In many applications the difference in the predicted system dynamics for these two conditions should be rather small. In the simulation example of this study, for example, damping needed to be increased significantly above established practice for this application to provoke a clear difference in the system dynamics for conditions $\mathrm{C}$ and $\mathrm{D}$.

The drawback of condition D compared with condition $\mathrm{C}$ is a higher computational cost since the behaviour of the contact force element itself also needs to be calculated. In, for example, optimization studies, where the system dynamics needs to be predicted for a high number of design parameter combinations, this should be considered [14].

In summary, transition condition $\mathrm{D}$ is the most general and, principally, the correct condition together with this contact force element. However, if the computational cost is crucial, it should be investigated whether there are special circumstances that make the use of transition condition $\mathrm{C}$ possible.

\section{ACKNOWLEDGEMENTS}

Financial support from the Swedish Knowledge Foundation and the Blekinge Institute of Technology is gratefully acknowledged. 


\section{REFERENCES}

1 Szabó, I. Geschichte der mechanischen Prinzipen und ihrer wichtigsten Anwendungen, 1977, pp. 425-479 (Birkhäuser Verlag, Basel).

2 Tateyama, K., Nakajima, S., and Fujiyama, T. The evaluation of ground properties and its application to the automatic control of vibratory soil compactors. In Proceedings of 12th International Symposium on Automation and Robotics in Construction (ISARC), Warsaw, 30 May-1 June 1995, pp. 563-570.

3 Natsiavas, S. and Gonzalez, H. Vibration of harmonically excited oscillators with asymmetric constraints. J. Appl. Mechanics, 1992, 59, 284-290.

4 Natsiavas, S. Dynamics of multiple-degree-of-freedom oscillators with colliding components. J. Sound Vibr., 1993, 165(3), 439-453.

5 Jerrelind, J. and Stensson, A. Braille printer dynamics. In Proceedings of 17th Biennial Conference on Mechanical Vibration and Noise, Las Vegas, 12-16 September 1999, paper DETC99/VIB-8032.

6 Fritzer, A. Nichtlineare Dynamik von Steuertrieben. VDI-Fortschrittberichte Schwingungstechnik, 1992, Ser. 11(176) (VDI-Verlag, Düsseldorf).

7 Pfeiffer, F. and Glocker C. Multibody dynamics with unilateral contacts, 1996 (John Wiley, New York).

8 Tateyama, K., Fujiyama, T., and Nishitani M. Aspects of the vibrating behavior of a vibratory roller in soil compaction. In Proceedings of 7th European International Society for Terrain-Vehicle Systems Conference, Ferrara, 8-10 October 1995.

9 Tateyama, K. and Fujiyama, T. Study on the vibratory roller-ground interaction and its application to the control of a roller. In Proceedings of 5th AsiaPacific Regional Conference International Society for Terrain-Vehicle Systems, Seoul, 20-22 October 1998, pp. 202-209.

10 Leine, R. I., van Campen, D. H., and van de Vrande, B. L. Bifurcations in nonlinear discontinous systems. Nonlinear Dynamics, 2000, 23, 105-164.

11 Modelica Standard Library Version 1.5, 1999 (ElastoGap), www.modelica.org.
12 Pietzsch, D. and Poppy, W. Simulation of soil compaction with vibratory rollers. J. Terramechanics, 1992, 29(6), 585-597.

13 Broman, G. and Jönsson, A. The nonlinear behaviour of a rammer soil compaction tamping machine. In Proceedings of 14th ASCE Engineering Mechanics Conference, Austin, Texas, 21-24 May 2000.

14 Broman, G., Jönsson, A., Englund, T., and Wall, J. Introductory optimisation study of a rammer soil compactor machine. In Proceedings of NAFEMS World Congress, 24-28 April 2001, pp. 395-406.

15 Anderegg, R. Nichtlineare Schwingungen bei dynamischen Bodenverdichtern. VDI-Fortschrittberichte, 1998, Ser. 4(146) (VDI-Verlag, Düsseldorf).

16 Yoo, T.-S. and Seelig, E. T. Dynamics of vibratory-roller compaction. J. Geotech. Engng Div., 1979, 105(10), $1211-1231$.

17 MATLAB ${ }^{\circledast}$ 6.5.1, 4 August 2003 (The MathWorks, Inc., Natick, Massachusetts).

18 Richart Jr, F. E., Hall Jr, J. R., and Woods, R. D. Vibration of soils and foundations, 1970 (PrenticeHall, Englewood Cliffs, New Jersey).

\section{APPENDIX}

\section{Notation}

$c \quad$ viscous damping $(\mathrm{N} \mathrm{s} / \mathrm{m})$

$d \quad$ distance (m)

$D \quad$ damping coefficient

$F$ force (N)

$g \quad$ constant of gravity $\left(\mathrm{m} / \mathrm{s}^{2}\right)$

$k \quad$ spring stiffness $(\mathrm{N} / \mathrm{m})$

$m$ mass $(\mathrm{kg})$

$t$ time (s)

$x \quad$ distance $(\mathrm{m})$

$\omega$

angular frequency $(\mathrm{rad} / \mathrm{s})$ 